\title{
Black-Burst-Based Multihop Broadcast Protocols for Vehicular Networks
}

\author{
Gökhan Korkmaz, Member, IEEE, Eylem Ekici, Member, IEEE, and Füsun Özgüner, Member, IEEE
}

\begin{abstract}
In this paper, two IEEE 802.11-based multihop broadcast protocols, namely urban multihop broadcast and ad hoc multihop broadcast, are proposed to address the broadcast storm, hidden node, and reliability problems of multihop broadcast in vehicular networks. In the proposed protocols, the functions of forwarding and acknowledging the broadcast packet are assigned to only one vehicle by dividing the road portion inside the transmission range into segments and choosing the vehicle in the furthest nonempty segment without a priori topology information. The simulation results confirm that our protocols have very high success rate and efficient channel utilization when compared with other flooding-based protocols. It is also concluded that there is no need for infrastructure support unless the line of sight among different road segments incident to an intersection is blocked with obstacles.
\end{abstract}

Index Terms-Communication standards, communication systems, protocols, road vehicles, transportation, wireless LAN.

\section{INTRODUCTION}

$\mathbf{R}$ ECENTLY, intervehicular communication (IVC) systems have attracted considerable attention from the research community and the automotive industry [1]. Many automobile manufacturers started planning to build communication devices into their vehicles for the purposes of safety, comfortable driving, and entertainment. Although the importance and possible applications of vehicle-to-vehicle and vehicle-toinfrastructure communications are enormous, this technology is in its initial stages. As an important milestone in October 1999, the Federal Communications Commission allocated the dedicated short-range communication (DSRC) spectrum at $5.9 \mathrm{GHz}$ "to increase traveler safety, reduce fuel consumption and pollution, and continue to advance the nation's economy" [3]. DSRC employs a standard that is similar to IEEE 802.11.

In IVC systems, broadcast is a frequently used method. Possible applications relying on broadcast include sharing emergency, traffic, weather, and road data among vehicles and delivering advertizements and announcements. These applications generate packets of various lengths at different rates. For example, accident warnings are short packets that are generated infrequently. Warning packets reporting slippery road

Manuscript received November 15, 2005; revised July 17, 2006 and October 15, 2006. This work was supported by OKI Electric Ind. Co. Ltd. The review of this paper was coordinated by Prof. C. Lin.

G. Korkmaz was with the Department of Electrical and Computer Engineering, The Ohio State University, Columbus, OH 43210 USA. He is now with ArrayComm, San Jose, CA 95131 USA (e-mail: korkmazg@ece.osu.edu).

E. Ekici and F. Özgüner are with the Department of Electrical and Computer Engineering, The Ohio State University, Columbus, OH 43210 USA (e-mail: ekici@ece.osu.edu; ozguner@ece.osu.edu).

Digital Object Identifier 10.1109/TVT.2007.900493 conditions are generally short, but these packets may be sent in bursts. Finally, advertisement packets of restaurants or hotels can be broadcast in very long packets that carry pictures, directions, or even short videos.

When a message is disseminated to locations beyond the transmission range, multihopping is used. Unfortunately, interference, packet collisions, and hidden nodes can stop the message dissemination during multihop broadcast. Moreover, multihop broadcast can consume a significant amount of wireless resources because of unnecessary retransmissions. These facts are important motivations for us to design a special medium access control (MAC) layer for efficient and reliable multihop message dissemination in vehicular ad hoc networks (VANETs) because high mobility and high number of nodes make multihop broadcast much more difficult in VANET environment. In [2], Xu et al. presented the simulation data to assess the feasibility of the communications component of cooperative active safety systems using IEEE 802.11a technology in the DSRC spectrum. It is concluded that it is worth doing further research on communication protocol design that might utilize the channel more efficiently even for single-hop broadcast.

The topology and the node movement of an IVC network is constrained by roads. The resulting communication network is a special type of a mobile ad hoc network (MANET), where the mobility rate is high but movement direction and speeds are predictable. In MANETs, flooding the network blindly is the first approach to achieve broadcasting since flooding can operate without local or global topology information. However, it has been shown in [3] that serious redundancy, contention, and collision problems occur as a result of flooding. Although [3] proposes techniques to improve blind flooding, the proposed solutions are not effective for all ranges of node densities and packet loads. In [4], the demand-driven transmission (DDT) protocol adjusts the timing of rebroadcast packets such that the vehicle furthest away from the source node retransmits earlier than the other nodes. In DDT, not all nodes retransmit the packet. If the uncovered area in a vehicle's transmission range after the previous transmissions is lower than a threshold, the vehicle does not retransmit the packet. Unfortunately, in IVC applications, both the node density and packet load fluctuate significantly, which makes the computation of optimum threshold value challenging and mostly infeasible. Moreover, the real uncovered area can be much higher than the theoretical uncovered area used in the protocol due to packet collisions and the varying wireless channel condition in time at different locations. In [5] and [6], methods to eliminate redundant packets while broadcasting is proposed using the topology information. Similarly, in the track detection protocol of [4], 
the neighborhood information is assumed to be available at every node. However, in an IVC network, the large number of vehicles and high mobility make such proactive approaches impractical [7].

Request to send/clear to send (RTS/CTS) handshake and acknowledgement (ACK) mechanisms are some of the methods that make the IEEE 802.11 [8] a widely accepted wireless local area network standard for point-to-point communication. The RTS/CTS mechanism decreases the effect of the hidden node problem, whereas the ACK mechanism makes the protocol reliable. However, since broadcast packets have more than one destination, employing RTS/CTS and ACK packets may cause packet storms around the source. To handle this problem, some protocols use the topology information to directly select the nodes that will send CTS and ACK packets [9], [10].

In [7], the IEEE 802.11 protocol is adapted for broadcasting in IVC systems by employing a distance-based waiting approach before retransmissions. Although this approach distributes the highly correlated rebroadcast times, problems such as hidden nodes, collisions at high packet traffic rates, reliability, and broadcast storms still persist. Another flooding-based protocol is proposed in [11] for broadcasting short packets in IVC systems. This protocol limits the channel access rate of each vehicle by defining a transmission window.

In this paper, we propose efficient IEEE 802.11-based multihop broadcast protocols, namely ad hoc multihop broadcast (AMB) and urban multihop broadcast (UMB) [12], for vehicular networks. Our proposed protocols are designed to address the broadcast storm, hidden node, and reliability problems in multihop broadcast. AMB and UMB protocols are composed of two phases, namely directional broadcast and intersection broadcast. We first introduce a new directional broadcast method, where sender nodes try to select the furthest node in the broadcast direction to assign the function of forwarding and acknowledging the packet without any a priori topology information, that is, senders select the furthest node without knowing the identification (ID) or position of their neighbors. The second phase, i.e., intersection broadcast, handles branching the packet dissemination at intersections. Although AMB and UMB protocols utilize the same directional broadcast mechanism, they differ in intersection broadcast. At the intersections where the communication among incident road segments are blocked by buildings, our UMB employs repeaters installed at intersections to forward the packet to all road segments. When the communication among road segments is not obstructed, there is no need for an infrastructure support, and packet branching is handled by an ad hoc algorithm using our AMB protocol.

\section{Directional BRoAdcAST}

The directional broadcast mechanism is the basis of both the AMB and UMB protocols. The difference between two protocols appears in handling intersections.

\section{A. Request to Broadcast/Clear to Broadcast (RTB/CTB) Handshake}

To avoid the hidden node problem while minimizing the overhead, sender vehicles engage in RTS/CTS handshake with only one of the recipients among the sender's neighbors. If we can select the furthest away node with RTS/CTS packets, then other nodes in between can overhear the transmission as well. To pick this vehicle, the protocol divides the road portion inside the transmission range into segments. Note that these segments are created only in the direction of dissemination. If there is more than one node in the furthest nonempty segment, this segment is divided iteratively into subsegments with smaller widths. If these segment-based iterations are not sufficient to pick only one node, the nodes in the last subsegment enter a random phase.

We refer to RTS and CTS as $R T B$ and $C T B$, respectively. In an RTB packet, in addition to the transmission duration, the source node includes its position and intended broadcast direction. If the source wants to disseminate the message in more than one direction, a new RTB packet should be generated for each direction.

1) First RTB Attempt: The source vehicle obeys all IEEE 802.11 transmission rules (carrier sense multiple access with collision avoidance) while attempting to send an RTB packet.

When the nodes in the direction of the dissemination receive this RTB packet, they compute their distance to the source node. Based on this distance, they send an energy burst (channel jamming signal), which is called black-burst. The black-burst method was proposed in [13] and [14] to provide guaranteed access delays to rate-limited packet traffic. In these proposals, the length of the original black-burst is proportional to the time that the node has been waiting for channel access. In our directional broadcast, we use the black-burst to select the furthest node by letting receivers sending black-burst signals proportional to their distance to the source. Since the position information of all nodes is unique, using the position information to determine the length of the black-burst gives us the capability of selecting the furthest node.

The duration of the black-burst signal in the first iteration is computed as follows:

$$
L_{1}=\left\lfloor d \cdot \frac{N_{\max }}{R}\right\rfloor \cdot \text { SlotTime }
$$

where $L_{1}$ is the black-burst duration in the first iteration, $d$ is the distance between the source and the vehicle, $R$ is the transmission range, $N_{\max }$ is the number of segments created, and SlotTime is the length of one slot. Note that $R / N_{\max }$ is the segment width and $\left\lfloor d *\left(N_{\max } / R\right)\right\rfloor$ is the number of slots the black-burst will keep busy. As a result of this computation, the furthest node sends the longest black-burst.

Nodes send their black-burst in the shortest possible time [i.e., short interframe space (SIFS)] after they hear the RTB packet. At the end of the black-burst, nodes turn around and listen to the channel. If they find the channel empty, it means that their black-burst was the longest, and they are now responsible for replying with a CTB packet after a duration called CTBTIME, where SIFS $<$ CTBTIME $<$ distributed interframe space. If they find the channel busy, it means that there are some other vehicles further away, and they do not try to send the CTB packet. 


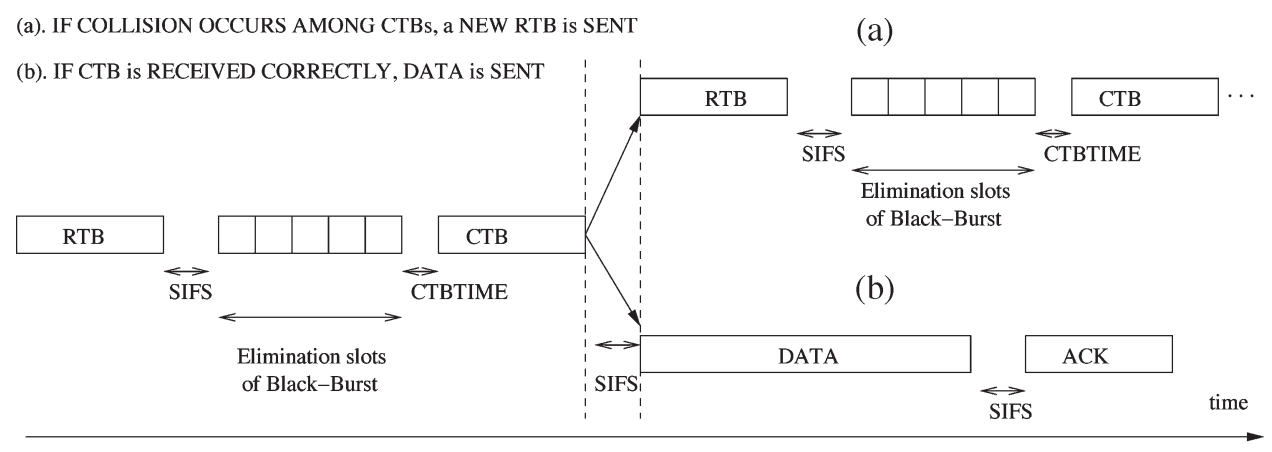

Fig. 1. Sequence of packets. (a) Second RTB/CTB handshake. (b) DATA/ACK.

2) Collision Among CTB Packets: When there is more than one vehicle in the furthest nonempty segment, they all find the channel empty after sending their black-bursts and continue to send CTB packets. However, since all vehicles start sending the CTB packets at the same time, their CTB packets will collide. When the source node detects a transmission but cannot decode the CTB packet, it detects the collision and repeats the RTB packet after SIFS time, as shown in Fig. 1(a). This time, only the nodes that have sent CTB packets join the collision resolution. To pick only one node, the furthest nonempty segment is divided into $N_{\max }$ subsegments. This process continues iteratively until a successful CTB packet is received by the source or until $D_{\max }$ iterations are completed. The length of the black-burst for the $i$ th iteration, i.e., $L_{i}$, is computed as follows:

$$
\begin{aligned}
L_{i} & \left.=\left\lfloor\left(d-L_{i-1}^{\text {longest }} \cdot W_{i-1}\right) \cdot \frac{N_{\max }}{W_{i-1}}\right)\right\rfloor \cdot \text { SlotTime } \\
W_{i} & =\frac{R}{N_{\max }^{i}}
\end{aligned}
$$

where $W_{i}$ is the segment width, and $L_{i}^{\text {longest }}$ is the length of the longest black-burst transmitted in the $i$ th iteration. In iteration i, $i \geq 2$, only vehicles that transmit CTB packets (i.e., that sent the longest black-burst) in iteration $i-1$ participate in contention resolution. The vehicles that participate in iteration $\mathrm{i}, i \geq 2$, simply use their black-burst length of the previous iteration, i.e., $L_{i-1}$, for $L_{i-1}^{\text {longest }}$.

Note that in an RTB packet, the source only indicates that there has been a collision: It is the receiver nodes' responsibility to choose the segment to be split. Only nodes that have sent the longest black-burst in the previous $(i-1)$ th iteration can join the current ( $i$ th) iteration. As a result, $L_{i-1}^{\text {longest }}$ is the black-burst length of these nodes who sent CTB in the previous iteration, and $L_{i-1}^{\text {longest }}+1$ is the segment to be split.

Our directional broadcast mechanism selects the corresponding node very quickly because it decreases the subsegment length very fast. As an example, when we divide the 400-m transmission range into ten segments, the subsegment length becomes only $4 \mathrm{~m}$ in the second iteration, where it is unlikely to find more than one vehicle.

3) Random Black-Burst Length: If the segment-based black-burst cannot resolve the collision after the $D_{\max }$ th iteration, the vehicles that have sent the CTB response in the last iteration enter the random collision resolution phase. In this phase, vehicles choose random black-burst lengths from $\left[0, N_{\max }-1\right]$ slots. When there is a collision, nodes whose CTBs have collided will choose another random number. If the source cannot get a successful CTB after $R a n_{\max }$ random iterations, it waits for a random amount of time and tries the segment-based collision resolution from the beginning. Starting the node selection process from the beginning can happen at most $R E T_{\max }$ number of times. The segment-based iterations decrease the segment to a very short strip after $D_{\max }$ iterations. As a result, only a small number of nodes will be left at the beginning of the random phase, and this will increase the success probability of this phase.

4) No Black-Burst Response: RTB packets may not be correctly received by other vehicles due to packet collisions. Detecting a free channel or failing to receive any CTB after sending the RTB packet, the source node assumes that no other vehicle has received its RTB packet. In this case, source node starts over with a new RTB packet after a random amount of time. Details of this backoff procedure are the same as those of the IEEE 802.11 standard when no CTS is received by the sender.

\section{B. Transmission of DATA and ACK}

After receiving a successful CTB, the source node sends its broadcast packet, as shown in Fig. 1(b). In this broadcast packet, the source node includes the ID of the node that has successfully sent the CTB. We will refer to this node as the corresponding node of the source. The corresponding node is now responsible for forwarding the broadcast packet and sending an ACK to the source. This ACK packet ensures the reliability of packet dissemination in the desired direction. Although all other nodes between the source and the ACK sender receive the broadcast packet, they do not rebroadcast or acknowledge it. If the ACK packet is not received by the source before the ACK timeout, the source goes back to the first segment-based iteration after a random amount of time. Details of this backoff procedure are the same as those of the IEEE 802.11 standard when ACK is not received. Note that there is a maximum number of times $R E T_{\max }$ that the source node can go back to the first iteration.

\section{INTERSECTION BROADCAST}

When there is an intersection in the path of the packet dissemination, new directional broadcasts should be initiated 
to all road directions at intersections. In this section, first, the UMB protocol is proposed to handle intersections where the line of sight is obstructed among road segments incident to the intersection. UMB protocol employs repeaters installed at the intersections where it has line of sight to all road segments. When the building obstruction is not a problem, a fully ad hoc intersection handling method, i.e., AMB, is proposed to eliminate the need for infrastructure support.

\section{A. $U M B$}

When a node is selected to forward a packet and it is outside the transmission range of a repeater, it continues with the directional broadcast protocol, as described in Section II. On the other hand, if the node is inside the transmission range of a repeater, the node sends the packet to the repeater using the point-to-point IEEE 802.11 protocol. Note that each node knows the locations of itself, intersections, and repeaters with the help of the Global Positioning System and digital road map. According to our protocol, a node sends RTS to the repeater, and only the repeater replies with the CTS packet if the channel is empty. Upon receiving the CTS packet from the repeater, the node sends the DATA packet, and the transmission ends when it receives an ACK packet from the repeater. After receiving this broadcast packet, the repeater initiates a directional broadcast in all road directions other than the direction where it received the packet from. In our UMB protocol, message dissemination is reliable as a result of the ACK mechanism; therefore, vehicles keep repeating after some random backoff time if they cannot receive a response from the repeater. However, in the worst case, if a vehicle fails to communicate with the repeater after $R E T_{\max }$ attempts, the vehicle changes the protocol operation to AMB and operates like a hunter vehicle, whose details are explained in Section III-B.

\section{B. Fully Ad Hoc Intersection Handling With the AMB Protocol}

The UMB protocol is an effective protocol for urban canyons with repeaters installed at the intersections. It is, however, not necessary to install repeaters at the intersections when the lineof-sight path exists among road segments. In this section, we propose a fully ad hoc extension to the UMB protocol, which handles intersections without infrastructure support when there is line of sight among all road segments. In the AMB protocol, a new intersection broadcast mechanism is proposed, where vehicles find the best candidate among themselves to branch the packet dissemination to other road segments. The vehicle closest to the intersection is a good candidate for this function because it is likely that vehicles closer to the intersection have a better coverage of the other road segments.

The new intersection broadcast mechanism is composed of two phases. The first phase is choosing a hunter vehicle, which tries to select the closest vehicle to the intersection. For this purpose, we will define an intersection region. In the second phase, the hunter vehicle initiates a search to find the closest vehicle, and in response to this search, vehicles reply with a black-burst according to their distance to the intersection. Once the hunter vehicle selects the closest vehicle to the intersection, this vehicle becomes responsible for branching the message to the other road segments.

1) Selecting the Hunter Vehicle: In the directional broadcast protocol, as described in Section II, the dissemination of messages is controlled by a subset of vehicles in the network. These vehicles are assigned the function of forwarding the message after the RTB/CTB handshake. Since each of these vehicles chooses a new vehicle in the transmission range $R$ to forward the message, at least one vehicle is chosen in every $R$ meters. Keeping this fact in mind, we have defined an intersection region around each intersection starting at $R / 2$ meters before and extending to $R / 2$ meters beyond the intersection. Note that at least one vehicle is chosen inside this region during the directional broadcast when the intersection region length is at least $R$ meters. The first vehicle chosen in the intersection region becomes the hunter vehicle.

Another reason to choose the intersection region starting at $R / 2$ meters before the intersection is as follows: Since the hunter vehicle tries to select a closer vehicle than itself, its transmission range should cover the points closer to the intersection than itself. When we use a transmission region with the proposed borders, in the worst case, the hunter vehicle is $R / 2$ meters away from the intersection, and it can cover the points up to $R / 2$ meters away at the other side of the intersection.

2) Selecting a Vehicle for Branching the Packet Dissemination: Having being selected inside the intersection region, the hunter vehicle sends an RTB packet that is different from the regular RTB, as defined in Section II-A. This new type of RTB packet that is employed to select the closest vehicle to the intersection is called intersection RTB (I-RTB). The black-burst response to an I-RTB is different from the response to a regular RTB that is employed in the directional broadcast; that is, when vehicles receive a regular RTB, the furthest vehicle from the source sends the longest black-burst. On the other hand, when vehicles receive an I-RTB, the vehicle closest to the intersection sends the longest black-burst.

The black-burst length for the I-RTB, i.e., $\hat{L}$, is calculated using (1) and (2). Note that these equations give the $L$ values for the regular RTB response. While determining the blackburst response length for an I-RTB, vehicles use a different $d$ parameter, i.e., $\hat{d}$, which is the distance from the intersection instead of the distance from the source. Thus

$$
\hat{d}=\sqrt{\left(X_{n}-X_{\mathrm{int}}\right)^{2}+\left(Y_{n}-Y_{\mathrm{int}}\right)^{2}}
$$

where $\left(X_{n}, Y_{n}\right)$ is the position of the node that sends the blackburst, and $\left(X_{\mathrm{int}}, Y_{\mathrm{int}}\right)$ is the position of the intersection.

Once this $L_{i}$ value is found by using $\hat{d}, \hat{L}$ is computed as follows:

$$
\hat{L}_{i}(\hat{d})=\left(N_{\max }-1\right)-L_{i}(\hat{d})
$$

The vehicle that sends the longest black-burst finds the channel idle and sends the CTB packet. After the transmission of the DATA and ACK packets, the selection process is finished. This 


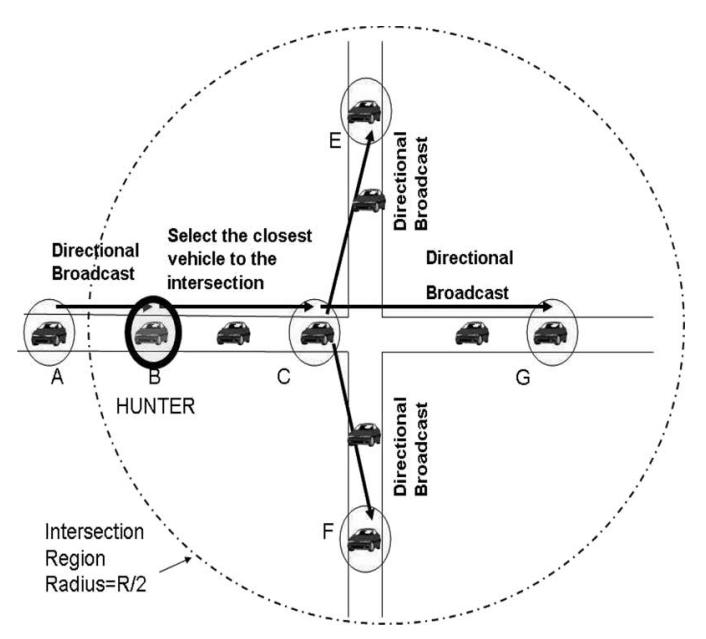

Fig. 2. Ad hoc intersection handling example.

selected vehicle becomes responsible for initiating directional broadcasts in all road directions, except the direction from which it received the packet.

An example of intersection handling is illustrated in Fig. 2. In this figure, since vehicle $\mathrm{A}$ is outside the intersection region, it uses the directional broadcast to reach vehicle B. Vehicle B is the first selected vehicle inside the intersection region; therefore, it becomes the hunter and initiates a vehicle selection process by sending the I-RTB packet. Being closest to the intersection, vehicle $\mathrm{C}$ sends the longest black-burst and then the CTB packet. After vehicle B assigns the function of branching the packet dissemination to vehicle $\mathrm{C}$, vehicle $\mathrm{C}$ initiates directional broadcasts to east, north, and south directions.

As a special case, if the hunter vehicle becomes unsuccessful in selecting a vehicle, the hunter vehicle itself becomes responsible for forwarding the packet to the other road segments.

\section{Branching Analysis}

In Section III-B1, we defined an intersection region whose length is $R$. In this section, we will see the effect of choosing a different intersection region length, such as $X$, as shown in Fig. 3. To successfully branch our packet dissemination in the north direction, we should have at least one car in region $\mathrm{X}$ and at least one car in region $\mathrm{Y}$. The successful branching probability is

$$
P_{\text {success }}=P[\text { at least one car in } \mathrm{X}] \cdot P[\text { at least one car in } \mathrm{Y}] \text {. }
$$

When we fix our intersection region length as $X$, the vehicle that will initiate a directional broadcast can be anywhere in $\mathrm{X}$. When this vehicle is right at the intersection point, it has the best coverage of the road segments. When this is the case, there is no difference among different lengths of intersection regions. In fact, this case applies to the UMB protocol since the repeater is located at this best possible location. In this analysis, the worst-case coverage will be investigated for the AMB protocol; therefore, we assume that the vehicle is at the

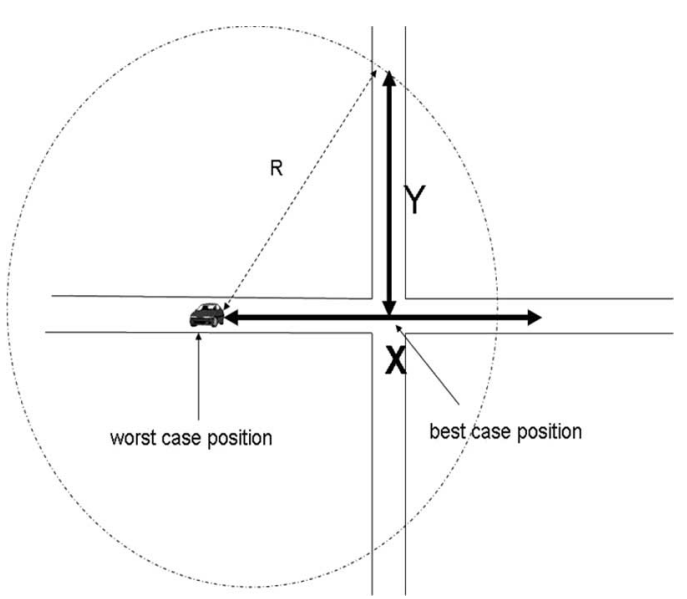

Fig. 3. Branching analysis. Simple intersection.

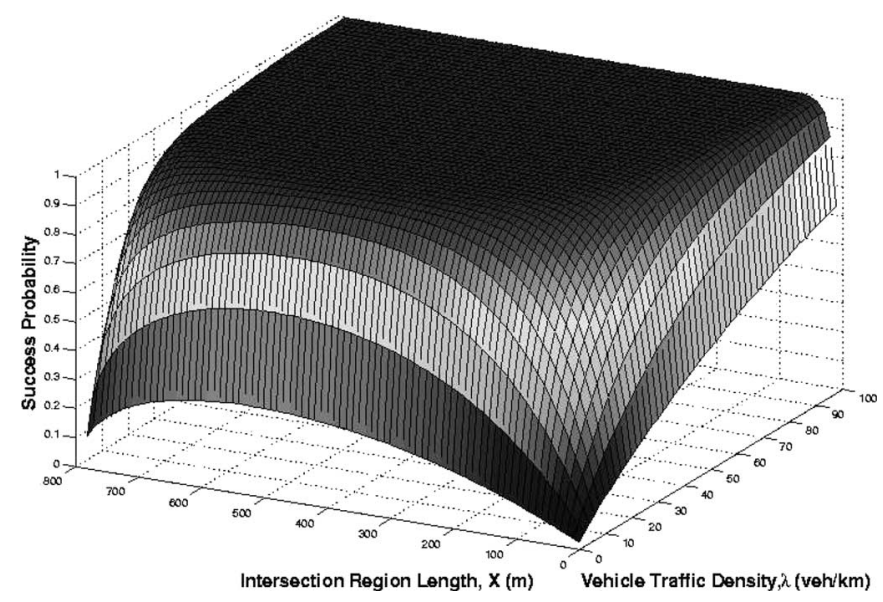

Fig. 4. Branching analysis. Success probability.

intersection region border. For a simple intersection, such as the one illustrated in Fig. 3, the shortest $Y$ is

$$
Y=\sqrt{R^{2}-\left(\frac{X}{2}\right)^{2}} .
$$

Since $P$ [at least one car in $\mathrm{X}]=1-P[$ no car in $\mathrm{X}]$, the successful branching probability of the AMB protocol in the worst case is

$$
P_{\text {success }}(X, L)=\left(1-e^{-\lambda \cdot X}\right) \cdot\left(1-e^{-\lambda \cdot \sqrt{R^{2}-\left(\frac{X}{2}\right)^{2}}}\right)
$$

where $\lambda$ is the average vehicle density. It is assumed that interspaces among vehicles are exponentially distributed.

Fig. 4 shows $P_{\text {success }}(X, \lambda)$. Vehicle density varies with time in an intersection; therefore, the chosen intersection region length $X$ should give a high $P_{\text {success }}$ for all vehicle densities. In Fig. 4, it can be observed that at $X=R=400 \mathrm{~m}$, the graph is close to the maximum for all $\lambda$ values, and the region around this point is almost flat. To quantify this observation, we have plotted the maximum success probability and the success probability for $X=R=400 \mathrm{~m}$ for a range of vehicle densities in Fig. 5. The figure shows that when $X=R$, the success probability is very close to the maximum for a wide range of vehicle densities. 


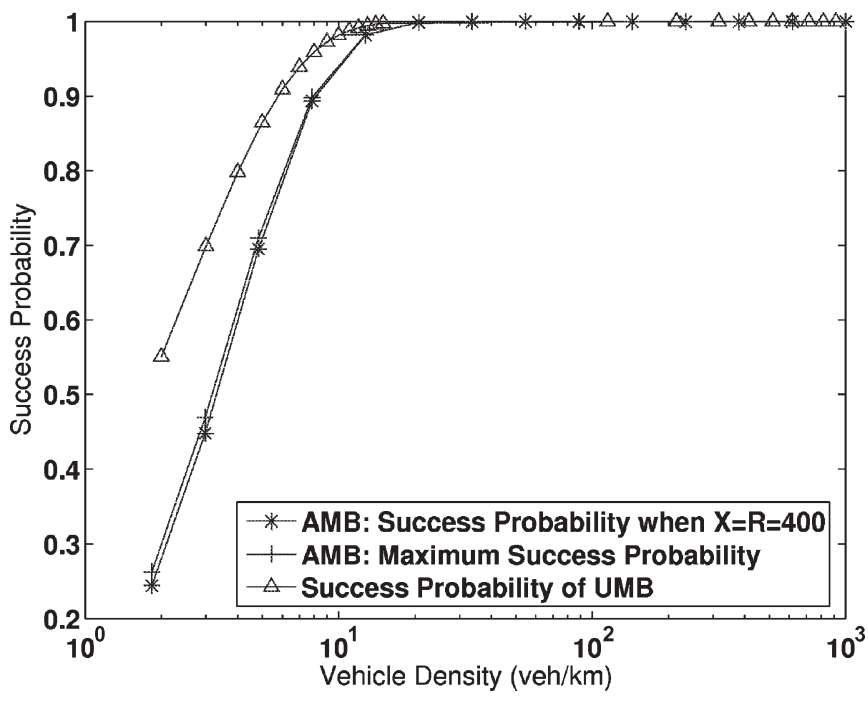

Fig. 5. Branching analysis. Success probability comparison.

Fig. 5 also includes the successful branching probability of the UMB protocol. Since there is a fixed repeater at the best position, the branching probability of UMB is

$$
P_{\mathrm{UMB}}=P[\text { at least one car in } \mathrm{Y}]=1-e^{-\lambda \cdot Y} .
$$

UMB's branching probability and AMB's worst-case branching probabilities are almost equal at high vehicle densities. The UMB protocol has higher success probability at low vehicle densities; however, this higher probability is at the cost of installing repeaters at every intersection. Furthermore, if the AMB protocol selects a node that is at the intersection, the success probability of the AMB and UMB protocol becomes equal at all traffic densities.

\section{Performance Evaluation}

To evaluate the performance of our AMB and UMB protocols, we have developed the wireless simulator using the eventdriven simulation library C-language-based simulator [15].

In addition to $\mathrm{AMB}$ and $\mathrm{UMB}$, we have simulated two more MAC layer protocols. In this paper, we will refer to these protocols as 802.11-distance and 802.11-random. They are flooding-based modifications of the IEEE 802.11 standard that route packets without the network topology information or any neighborhood knowledge. They try to avoid collisions among rebroadcast packets by forcing vehicles to wait before forwarding the packets. According to these protocols, every vehicle must broadcast every distinct packet.

The first protocol, namely 802.11-distance, employs the idea proposed in [7], where the waiting times of the vehicles are inversely proportional to their distances from the source. The waiting time $W T$ is computed as follows:

$$
W T=\left(-\left\lfloor\frac{\hat{d}}{\text { Range }} * \text { maxSlot }\right\rfloor+\text { maxSlot }\right) * \text { SlotTime }
$$

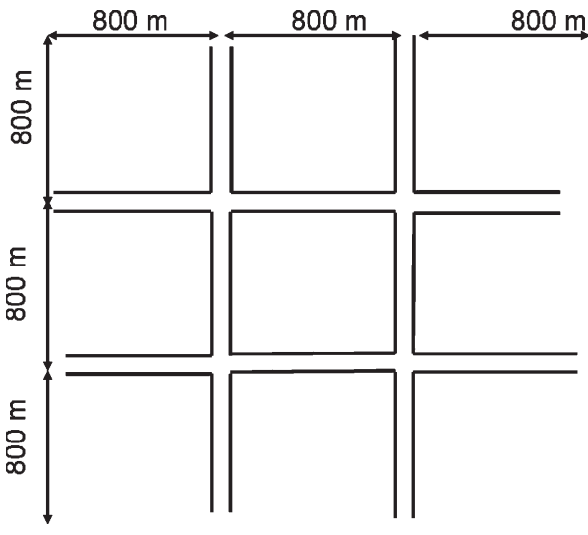

Fig. 6. Four intersections: $2400 \mathrm{~m} \times 2400 \mathrm{~m}$.

where maxSlot is the maximum possible number of slots a vehicle waits before forwarding the packet. As in the IEEE 802.11 standard, vehicles decrease their waiting time counters when they find the channel empty and freeze them when the channel is busy. The protocol proposed in [7] continuously computes the waiting time; however, in our 802.11-distance implementation, waiting times are discrete since all waiting times are computed as multiples of SlotTime in the IEEE 802.11 standard.

In the second protocol, namely 802.11-random, when a vehicle receives a broadcast packet, it will wait for a random duration $W T$ before forwarding the packet, i.e.,

$$
W T=\text { nSlots } * \text { SlotTime }
$$

where $n$ Slot is a random number between $[0$, max Slot $]$.

\section{A. Simulation Parameters}

Two types of road geometry is simulated in our scenarios. The first road geometry is the simple road geometry representing a highway segment of $3000 \mathrm{~m}$. The second road geometry has four intersections and is shown in Fig. 6. Each road segment contains two lanes: one for each direction of traffic flow. Initially, the vehicles are randomly placed on the road segments with exponentially distributed interspaces. Lane changes, turns, and overtaking are not modeled for vehicle movement. Each vehicle is assigned a speed from a Gaussian distribution with a mean of $40 \mathrm{~km} / \mathrm{h}$ and a standard deviation of $5 \mathrm{~km} / \mathrm{h}$ at the beginning of the simulation, and this speed remains constant during the simulation. The AMB and UMB protocols are simulated with the following parameters: $R E T_{\max }=15, N_{\max }=10, D_{\max }=3$, and $\operatorname{Ran}_{\max }=2$. The common parameters that are used for all protocols are as follows: vehicle density $=33 \mathrm{veh} / \mathrm{km}$ per lane, total number of vehicles $=619$, transmission range $=400 \mathrm{~m}$, data rate $=1 \mathrm{Mb} / \mathrm{s}$, frame body $=2304$ bytes or 100 bytes, base protocol $=802.11 \mathrm{~b}, \max S l o t=32$, SlotTime $=20 \mu \mathrm{s}$, simulation time $($ simtime $)=60 \mathrm{~s}$, and simulation repetitions $=$ 30 . The other parameters of the MAC layer and the physical layer are taken from the IEEE 802.11b standard document [16]. 


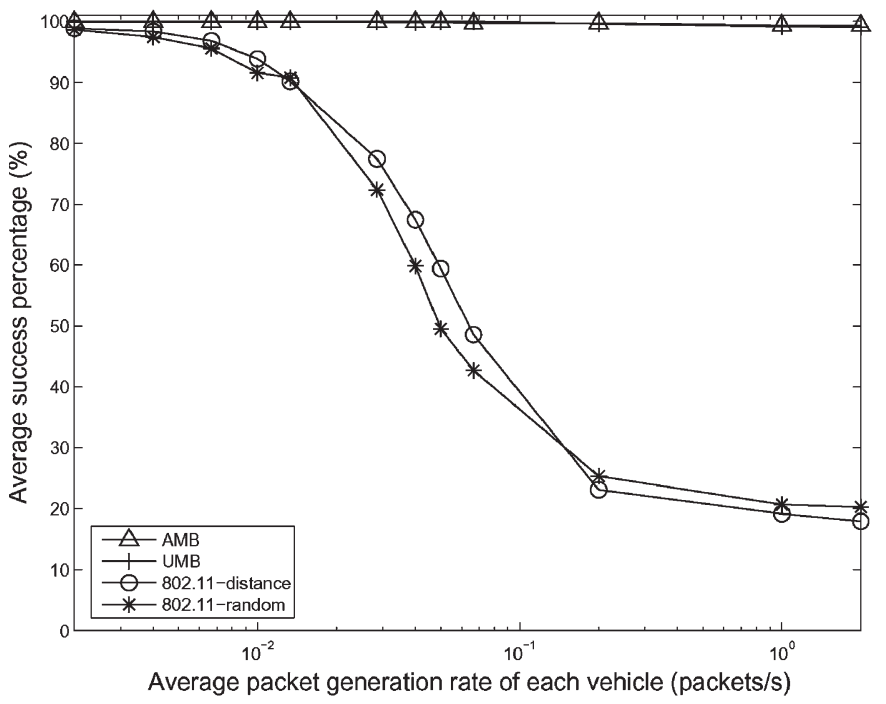

(a)

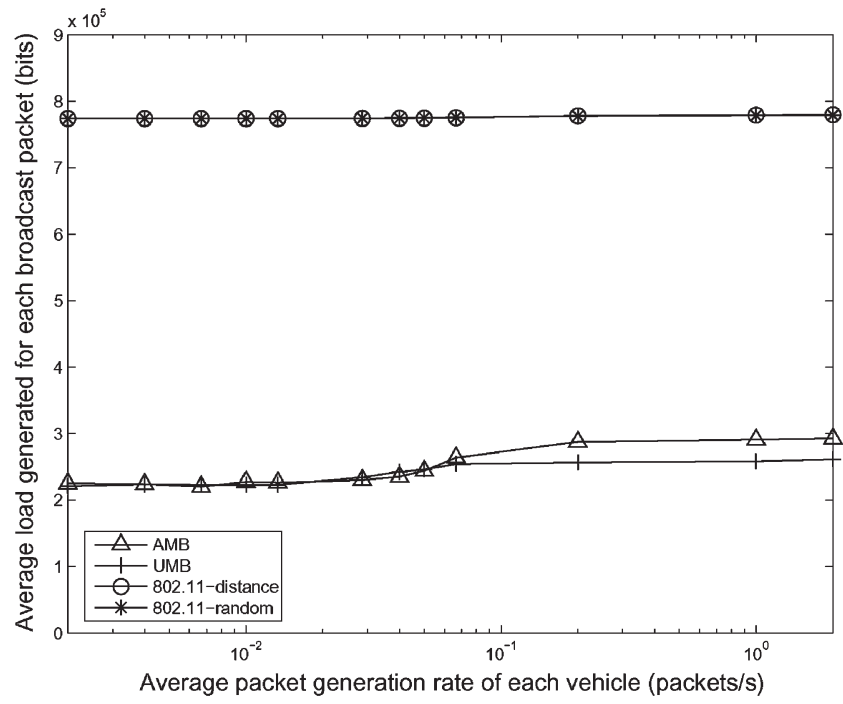

(c)

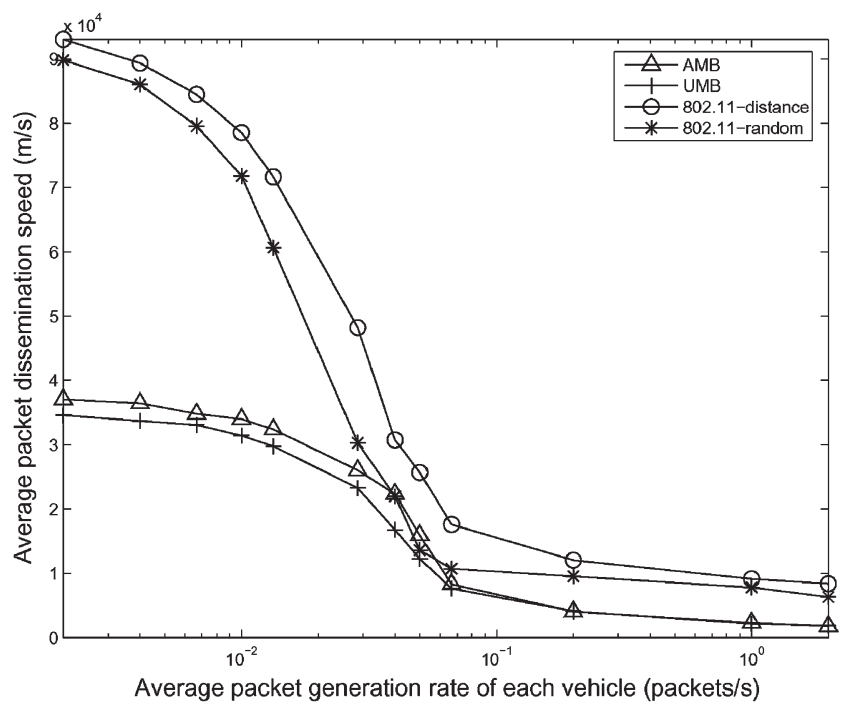

(e)

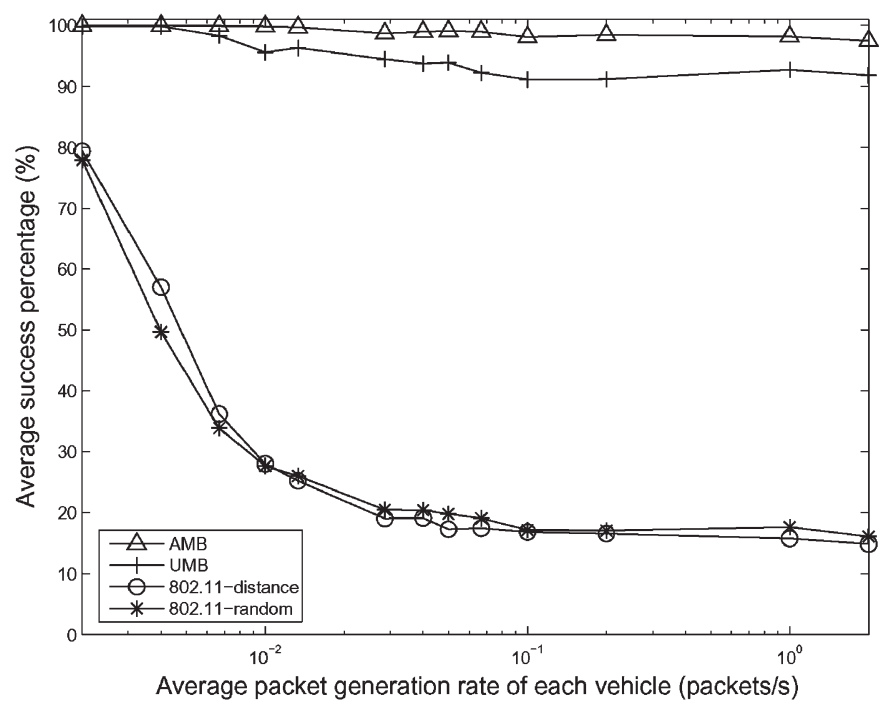

(b)

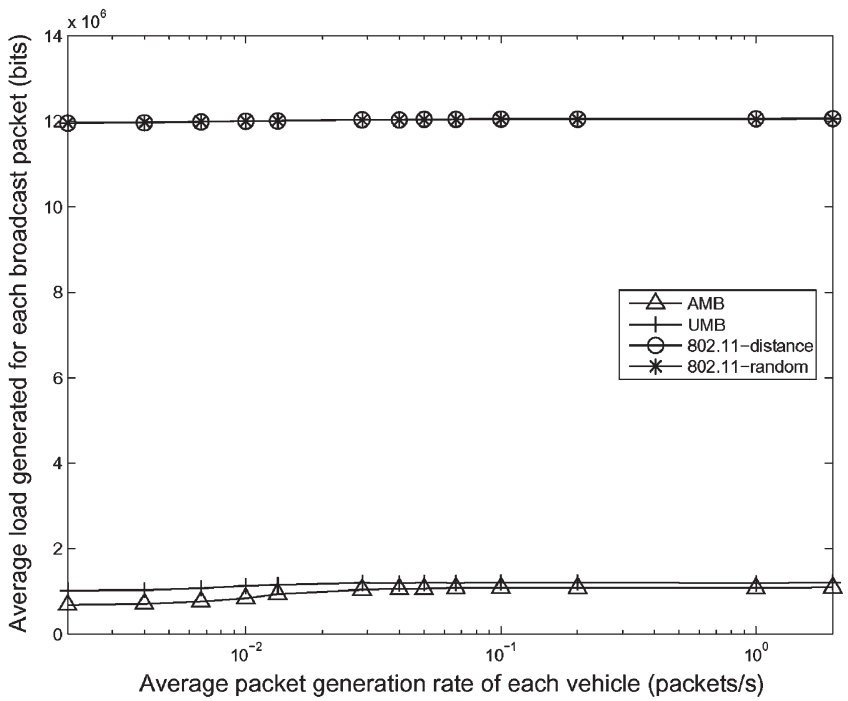

(d)

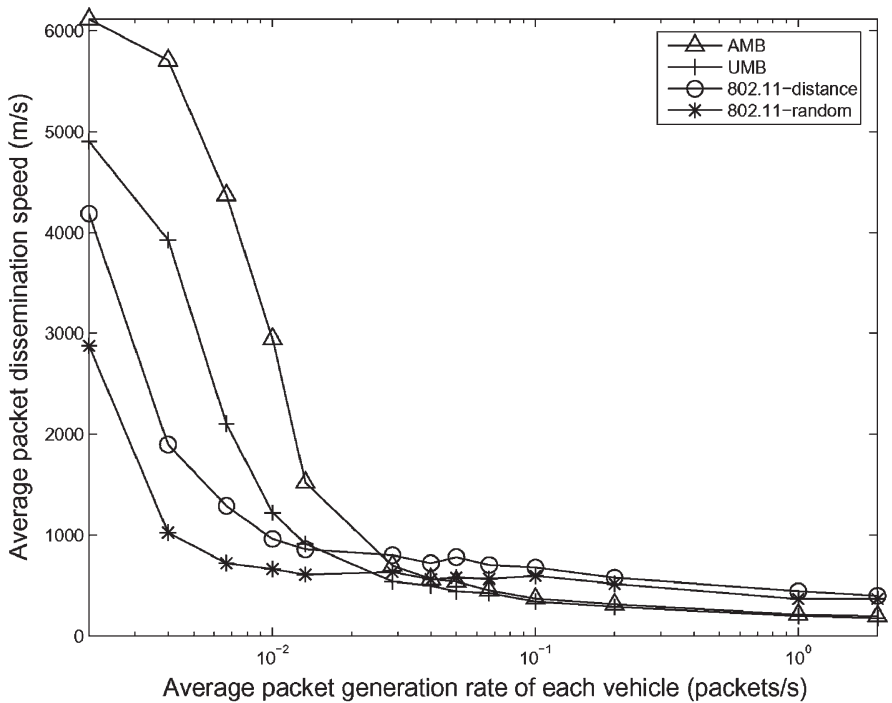

(f)

Fig. 7. Four intersections. Average vehicle density $=33.3$ veh $/ \mathrm{km}$ per lane. (a), (c), and (e) Payload is 100 bytes. (b), (d), and (f) Payload is 2304 bytes. (a) and (b) Successful packet delivery percentage. (c) and (d) Normalized average load per broadcast packet. (e) and (f) Packet dissemination speed. 


\section{B. Results}

In this section, we present only the results of the scenario with four intersections since space is limited, and the results are very similar to results of the highway scenario.

1) Successful Packet Delivery Percentage: The successful packet delivery percentage of a packet is the ratio of the cars that receive the broadcast packet to the total number of cars. When the average successful packet delivery ratio is lower than $100 \%$, it means that the broadcast packets are not received by all vehicles. Fig. 7(a) and (b) depicts the average successful packet delivery ratio when payload lengths of 100 and 2304 bytes are used, respectively. It shows that our AMB protocol achieves approximately $100 \%$ successful packet delivery. The AMB protocol has a higher successful delivery ratio than that of the UMB protocol, particularly when the payload is long [Fig. 7(b)], because the UMB protocol relies on just one node, i.e., the repeater, at the intersection that creates a hot spot. This single hot spot increases packet collisions and queuing delays. On the other hand, the AMB protocol chooses different vehicles at the intersection to initiate packet dissemination to other road segments. Similar to the highway scenario, 802.11-distance and 802.11-random protocols perform poorly because of packet collisions due to hidden nodes and the lack of the acknowledgment mechanism.

2) Normalized Load Generated per Broadcast Packet: The load generated per broadcast packet is the total number of bits transmitted to disseminate a packet in all retransmissions. To compute the average load, we divide the total number of bits sent by the total number of broadcast packets generated during simulation. This metric gives the total traffic generated by one broadcast packet in the network. Note that small values of this parameter correspond to efficient usage of the channel. When the dissemination of a packet stops, it can reach only a fraction of the network, and it generates a smaller load as compared with a packet that reaches all vehicles. For a fair comparison, we divide the load generated by the successful packet delivery percentage and define a normalized metric for the average load generated per broadcast packet. Fig. 7(c) and (d) shows the normalized average load generated per broadcast packet. For both payload lengths, we can observe that the AMB and UMB protocols generate less load while disseminating a packet as compared with the 802.11-random and 802.11-distance protocols because AMB and UMB protocols assign the function of forwarding the broadcast packet to only one vehicle, whereas flooding-based protocols assign this function to every vehicle. For the short payload, AMB and UMB generate approximately 2.5 times less load than the flooding protocols. Since the length of the control packets (RTB, CTB, ACK, and black-burst) becomes negligible when compared with the length of the DATA packet in Fig. 7(d), the AMB and UMB protocols perform approximately 12 times better than the flooding protocols.

3) Packet Dissemination Speed (in Meters per Second): The speed of a packet at a point is computed by dividing the distance traveled by the packet by the delay. In the context of this paper, delay refers to the time elapsed between the instant the packet enters the source queue and the reception time of the packet by another vehicle. Fig. 7(e) and (f) shows the packet dissem- ination speed for short and long DATA packets, respectively. Fig. 7(e) depicts that when the DATA packet length is short, 802.11-distance and 802.11-random disseminate the packets faster than the AMB and UMB protocols. However, recall that the successful packet delivery percentages of these protocols are extremely low, as shown in Fig. 7(b). This low packet delivery percentage decreases the queuing delays of 802.11distance and 802.11-random. Increasing the length of DATA packet increases the speed of the AMB and UMB protocols relative to the other protocols. In Fig. 7(f), AMB becomes the fastest one at low packet generation rates, and its speed is close to the others at high packet generation rates, in spite of the fact that 802.11-distance and 802.11-random protocols drop many packets.

\section{CONCLUSiON}

In this paper, two position-based multihop broadcast protocols for IVC networks have been proposed. The difference between the two protocols is their method of handling intersections. When the line-of-sight communication among the road segments incident to an intersection is obstructed, the UMB protocol employs repeaters installed at the intersections. The second protocol, namely AMB, is an extension of the UMB protocol with a fully ad hoc intersection broadcast mechanism. It is shown through simulations that when the communication among the different road segments incident to the intersection is not blocked with obstacles, our AMB protocol has a very high successful packet delivery ratio and efficient channel usage, even at high packet loads; therefore, there is no need for an infrastructure support. It is concluded that the best solution for the broadcast problem in vehicular networks is using UMB and AMB protocols together, that is, the UMB protocol should be used to handle intersections where the communication among road segments requires a repeater because of buildings, and the AMB protocol should be used to handle intersections without repeaters. In our future work, the performance of the UMB and AMB protocols will be improved using directional antennas.

\section{REFERENCES}

[1] H. Hartenstein, B. Bochow, A. Ebner, M. Lott, M. Radimirsch, and D. Vollmer, "Position-aware ad hoc wireless networks for inter-vehicle communications: The fleenet project," in Proc. ACM Symp. MobiHOC, Oct. 2001.

[2] Q. Xu, T. Mak, J. Ko, and R. Sengupta, "Vehicle-vehicle safety messaging in DSRC," in Proc. 1st ACM Workshop Veh. Ad-hoc Netw., Oct. 2004, pp. 19-28.

[3] Y.-C. Tseng, S.-Y. Ni, Y.-S. Chen, and J.-P. Sheu, "The broadcast storm problem in a mobile ad hoc network," Wirel. Netw., vol. 8, no. 2/3, pp. 153-167, Mar.-May 2002.

[4] M.-T. Sun, W.-C. Feng, T.-H. Lai, K. Yamada, H. Okada, and K. Fujimura, "GPS-based message broadcast for adaptive inter-vehicle communications," in Proc. VTC, 2000, vol. 6, pp. 2685-2692.

[5] W. Lou and J. Wu, "On reducing broadcast redundancy in ad hoc wireless networks," IEEE Trans. Mobile Comput., vol. 1, no. 2, pp. 111-122, Apr.-Jun. 2002.

[6] I. Stojmenovic, M. Seddigh, and J. Zunic, "Dominating sets and neighbor elimination based broadcasting algorithms in wireless networks," IEEE Trans. Parallel Distrib. Syst., vol. 13, no. 1, pp. 14-25, Jan. 2002.

[7] L. Briesemeister and G. Hommel, "Role-based multicast in highly mobile but sparsely connected ad hoc networks," in Proc. IEEE/ACM Workshop MobiHOC, Boston, MA, Aug. 2000, pp. 45-50. 
[8] The Institute of Electrical and Electronics Enginneers (IEEE), Wireless LAN Medium Access Control (MAC) and Physical Layer (PHY) Specifications, (a.k.a. ISO/IEC 8802-11:1999(E)), ANSI/IEEE Std.802.11, 1999. [Online]. Available: http://standards.ieee.org

[9] K. Tang and M. Gerla, "MAC reliable broadcast in ad-hoc networks," in Proc. MILCOM, Oct. 2001, vol. 2, pp. 28-31.

[10] M.-T. Sun, L. Huang, A. Arora, and T.-H. Lai, "Reliable MAC layer multicast in IEEE 802.11 wireless networks," in Proc. Int. Conf. Parallel Process., 2002, pp. 527-536.

[11] K. Tokuda, M. Akiyama, and H. Fujii, "DOLPHIN for inter-vehicle communications system," in Proc. IEEE Intell. Veh. Symp., 2000, pp. 504-509.

[12] G. Korkmaz, E. Ekici, F. Ozguner, and U. Ozguner, "Urban multi-hop broadcast protocols for inter-vehicle communication systems," in Proc. 1st ACM Workshop VANET, 2004, pp. 76-85.

[13] J. L. Sobrinho and A. S. Krisnakumar, "Distributed multiple access procedures to provide voice communications over IEEE 802.11 wireless networks," in Proc. Global Telecommun. Conf., 1996, vol. 3, pp. 1689-1694.

[14] J. L. Sobrinho and A. S. Krisnakumar, "Quality-of-service in ad-hoc carrier sense multiple access wireless networks," IEEE J. Sel. Areas Commun., vol. 17, no. 8, pp. 1353-1368, Aug. 1999.

[15] H. Schwetman, "CSIM User's Guide Rev. 1," Microelectron. and Comput. Technol. Corporation, Tech. Rep. ACT-126-90, 1991. Rev. 1.

[16] LAN/MAN Standards Committee of the IEEE Computer Society, Part11: Wireless LAN Medium Access Control (MAC) and Physical Layer (PHY) Specifications: Higher-Speed Physical Layer Extension in the $2.4 \mathrm{GHz}$ Band, IEEE Std. 802.11b-1999, 1999.

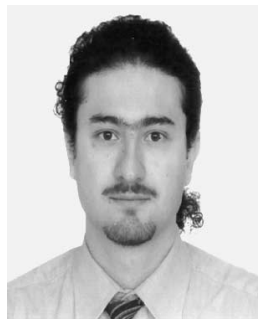

Gökhan Korkmaz (S'05-M'06) received the B.S. degree in electrical engineering from Middle East Technical University, Ankara, Turkey, in 2000 and the M.S. and Ph.D. degrees in electrical and computer engineering from The Ohio State University, Columbus, in 2002 and 2006, respectively.

Since October 2006, he has been a System Research Engineer with ArrayComm, San Jose, CA. His main research interests are wireless local area networks (IEEE 802.11), with special emphasis on vehicular communication systems and wireless metropolitan area networks (IEEE 802.16).

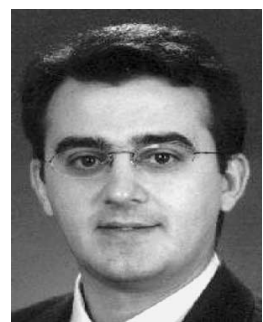

Eylem Ekici (S'99-M'02) received the B.S. and M.S. degrees in computer engineering from Bogazici University, Istanbul, Turkey, in 1997 and 1998, respectively, and the Ph.D. degree in electrical and computer engineering from the Georgia Institute of Technology, Atlanta, in 2002.

$\mathrm{He}$ is currently an Assistant Professor with the Department of Electrical and Computer Engineering, The Ohio State University, Columbus. His current research interests include wireless sensor networks, next-generation wireless systems, and vehicular communication systems, with a focus on routing and medium-access control protocols, resource management, and analysis of network architectures and protocols. He also conducts research on interfacing of dissimilar networks.

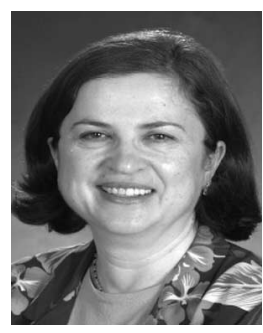

Füsun Özgüner (S'74-M'75) received the M.S degree in electrical engineering from Istanbul Technical University, Istanbul, Turkey, in 1972 and the $\mathrm{Ph} . \mathrm{D}$. degree in electrical engineering from the University of Illinois at Urbana-Champaign in 1975.

She was with the Design Automation Group, IBM T. J. Watson Research Center, Yorktown Heights, NY, for one year and then joined the faculty at the Department of Electrical Engineering, Istanbul Technical University, in 1976. Since January 1981, she has been with The Ohio State University (OSU) Columbus, where she is currently a Professor of electrical and computer engineering. She has also served as the Interim Department Chair of Electrical and Computer Engineering, OSU. Her current research interests are parallel and fault-tolerant architectures, heterogeneous distributed computing, real-time parallel computing and communication, and wireless networks.

Dr. Özgüner has served as an Associate Editor of the IEEE TRANSACTIONS ON COMPUTERS and on the program committees of several international conferences. 